Asia Pacific Journal of Mathematics, Vol. 5, No. 1 (2018), 50-59

ISSN 2357-2205

\title{
STABILITY AND OSCILLATION OF MIXED DIFFERENTIAL EQUATION WITH PIECEWISE CONTINUOUS ARGUMENTS
}

\author{
QI WANG*, XIAOMING WANG \\ School of Applied Mathematics, Guangdong University of Technology, Guangzhou, 510006, P.R. China \\ *Corresponding author: bmwzwq@126.com \\ Received Oct 5, 2017
}

\begin{abstract}
The paper focuses on the stability and oscillation of mixed differential equation with piecewise continuous arguments $u^{\prime}(t)=a u(t)+b u([t])+c u(2[(t+1) / 2])$. The explicit expression of the analytic solution, the conditions of stability and oscillation of the analytic solution are presented by the technique of solving differential equation, the theory of characteristic and the functional differential inequality, respectively. The results extend some existing ones.
\end{abstract}

2010 Mathematics Subject Classification.34D05; 34D23.

Key words and phrases. piecewise continuous arguments; analytic solution; stability; oscillation.

\section{INTRODUCTION}

Differential equation with piecewise continuous arguments (EPCA) is a special class of delay differential equations (DDEs), it has the properties of both the differential and difference equations in the structure, which makes it more effectively solve some problems that contain mixed system of continuous and discrete variables, such as feedback control [1]. EPCA has been initiated by Wiener [2], Shah and Wiener [3] and Cooke and Wiener [4]. In [5], Wiener extended and summarized his many research achievements of EPCA systematically.

In the past few decades, EPCA has received much attention from a number of investigators. In [6], the stability of the analytic solution of EPCA in the population model was studied. Alwan et al. [7] developed a comparison principle to establish some stability properties for nonlinear EPCA. In [8], the existence and uniqueness of almost periodic solution of EPCA were considered. Akhmet [9] discussed the asymptotic behavior of EPCA. In [10], the existence of integral manifolds of generalized type EPCA has been researched. For another population model with piecewise constant arguments, Muroya [11] constructed new contractivity condition. Bereketoglu et al.[12] obtained some conditions that guarantee the 
oscillation and asymptotic behaviour of the solutions of a third order nonlinear EPCA. Different from above cases, in our work, we shall study both stability and oscillation for a more complicated equation with scalar coefficients, and get some new results.

In the present paper we will consider the following mixed EPCA

$$
\begin{aligned}
& u^{\prime}(t)=a u(t)+b u([t])+c u\left(2\left[\frac{t+1}{2}\right]\right), \quad t \geq 0, \\
& u(0)=u_{0},
\end{aligned}
$$

where $a, b, c, u_{0}$ are real constants and [.] denotes the greatest integer function. Since the second argument deviation $t-2[(t+1) / 2]$ is negative in $[2 n-1,2 n)$ and positive in $[2 n, 2 n+1)$, so (1) is called as mixed type EPCA. In particularly, when $c=0$, the equation in (1) becomes $u^{\prime}(t)=a u(t)+b u([t])$, which is exactly the case of [13]. If let $b=0$, the equation in (1) becomes $u^{\prime}(t)=a u(t)+c u(2[(t+1) / 2])$, which is exactly the case of [14]. Thus, the results in this paper are the generalization of corresponding ones in [13] and [14].

\section{THE EXPLICIT EXPRESSION OF THE ANALYTIC SOLUTION}

Definition 1. [5] A solution of (1) on $[0, \infty)$ is a function $u(t)$ which satisfies the following conditions:

(i) $u(t)$ is continuous on $[0, \infty)$;

(ii) The derivative $u^{\prime}(t)$ exists at each point $t \in[0, \infty)$, with the possible exception of the points $[t] \in[0, \infty)$, where one-sided derivatives exist;

(iii) Eq. (1) is satisfied on each interval $[2 n-1,2 n) \in[0,+\infty)$ and $[2 n, 2 n+1) \in[0,+\infty)$ with integral end points.

Theorem 1. If $\lambda \neq 0$, then (1) has a unique solution on $[0, \infty)$

$$
u(t)=\left\{\begin{array}{l}
\left(\left(e^{a}+\frac{b+c}{a}\left(e^{a}-1\right)\right) e^{a(t-2 n+1)}+\frac{e^{a(t-2 n+1)}-1}{a}\left(b e^{a}+\frac{b(b+c)\left(e^{a}-1\right)}{a}+\lambda c\right)\right) \\
\lambda^{n-1} u_{0}, \quad t \in[2 n-1,2 n), \\
\left(e^{a(t-2 n)}+\frac{b+c}{a}\left(e^{a(t-2 n)}-1\right)\right) \lambda^{n} u_{0}, \quad t \in[2 n, 2 n+1),
\end{array}\right.
$$

for $a \neq 0$, where $n \in N$ and

$$
\lambda=\frac{\left(e^{a}+\frac{b}{a}\left(e^{a}-1\right)\right)\left(e^{a}+\frac{b+c}{a}\left(e^{a}-1\right)\right)}{1-\frac{c}{a}\left(e^{a}-1\right)},
$$

and

$$
u(t)=\left\{\begin{array}{l}
\left(\left(b^{2}+b c+b+\lambda c\right)(t-2 n+1)+b+c+1\right) \lambda^{n-1} u_{0}, \quad t \in[2 n-1,2 n) \\
((b+c)(t-2 n)+1) \lambda^{n} u_{0}, \quad t \in[2 n, 2 n+1),
\end{array}\right.
$$

for $a=0$, where $n \in N$ and

$$
\lambda=\frac{(b+1)(b+c+1)}{1-c} .
$$


Proof 1. Assume that $u_{n}(t)$ is a solution of (1) on the interval $[2 n-1,2 n+1)$ with conditions $u_{n}(2 n)=c_{2 n}$ and $u_{n}(2 n-1)=c_{2 n-1}$. So we have

$$
u_{n}^{\prime}(t)=a u_{n}(t)+b u_{n}(2 n-1)+c u_{n}(2 n)
$$

for $t \in[2 n-1,2 n)$ and

$$
u_{n}^{\prime}(t)=a u_{n}(t)+(b+c) u_{n}(2 n)
$$

for $t \in[2 n, 2 n+1)$, respectively.

For the first case, the general solution of (1) on the interval $[2 n-1,2 n)$ is

$$
u_{n}(t)=e^{a(t-2 n+1)} u_{n}(2 n-1)+\frac{1}{a}\left(e^{a(t-2 n+1)}-1\right)\left(b u_{n}(2 n-1)+c u_{n}(2 n)\right),
$$

let $t=2 n$, then (4) becomes

$$
u_{n}(2 n)=e^{a} u_{n}(2 n-1)+\frac{1}{a}\left(e^{a}-1\right)\left(b u_{n}(2 n-1)+c u_{n}(2 n)\right),
$$

which implies that

$$
c_{2 n}=e^{a} c_{2 n-1}+\frac{1}{a}\left(e^{a}-1\right)\left(b c_{2 n-1}+c c_{2 n}\right)
$$

hence

$$
c_{2 n}=\frac{e^{a}+b\left(e^{a}-1\right) / a}{1-c\left(e^{a}-1\right) / a} c_{2 n-1} .
$$

On the other hand, the general solution of (1) on the interval $[2 n, 2 n+1)$ is

$$
u_{n}(t)=\left(e^{a(t-2 n)}+\frac{b+c}{a}\left(e^{a(t-2 n)}-1\right)\right) u_{n}(2 n),
$$

let $t=2 n+1$, then (6) transforms into

$$
c_{2 n+1}=\left(e^{a}+\frac{b+c}{a}\left(e^{a}-1\right)\right) c_{2 n}
$$

Let

$$
\lambda=\frac{\left(e^{a}+b\left(e^{a}-1\right) / a\right)\left(e^{a}+(b+c)\left(e^{a}-1\right) / a\right)}{1-c\left(e^{a}-1\right) / a},
$$

then from (5) and (7) we obtain $c_{2 n+1}=\lambda c_{2 n-1}$. Therefore, the general solution of (1) is

$$
\begin{aligned}
u(t)= & \left(\left(e^{a}+\frac{b+c}{a}\left(e^{a}-1\right)\right) e^{a(t-2 n+1)}\right. \\
& \left.+\frac{e^{a(t-2 n+1)}-1}{a}\left(b e^{a}+\frac{b(b+c)\left(e^{a}-1\right)}{a}+\lambda c\right)\right) \lambda^{n-1} u_{0}
\end{aligned}
$$

for $t \in[2 n-1,2 n)$ and

$$
u(t)=\left(e^{a(t-2 n)}+\frac{b+c}{a}\left(e^{a(t-2 n)}-1\right)\right) \lambda^{n} u_{0}
$$

for $t \in[2 n, 2 n+1)$, respectively. 
The case of $a=0$ can be proved by letting $a \rightarrow 0$ in (2). The uniqueness of solution of (1) on $[0, \infty)$ follows from its continuity and uniqueness of solution on each interval $[2 n-1,2 n)$ and $[2 n, 2 n+1)$. The proof is completed.

Remark 1. In view of $\lambda \neq 0$ in Theorem 1. In the following we will consider the other cases except for the case of $\lambda \neq 0$. If $a \neq 0$ we have

(i) When $1-c\left(e^{a}-1\right) / a=0$ and $\left(e^{a}+b\left(e^{a}-1\right) / a\right)\left(e^{a}+(b+c)\left(e^{a}-1\right) / a\right) \neq 0$, it implies that $u(t)=0$.

(ii) When $1-c\left(e^{a}-1\right) / a=0$ and $\left(e^{a}+b\left(e^{a}-1\right) / a\right)\left(e^{a}+(b+c)\left(e^{a}-1\right) / a\right)=0$, (1) has infinitely many solutions.

(iii) When $1-c\left(e^{a}-1\right) / a \neq 0$ and $e^{a}+b\left(e^{a}-1\right) / a=0$ and $e^{a}+(b+c)\left(e^{a}-1\right) / a \neq 0$, the solution of (1) is

$$
u(t)=\left\{\begin{array}{l}
\left(e^{a t}+\frac{b+c}{a}\left(e^{a t}-1\right)\right) u_{0}, \quad t \in[0,1), \\
\left(e^{a(t-1)}+\frac{b}{a}\left(e^{a(t-1)}-1\right)\right)\left(e^{a}+\frac{b+c}{a}\left(e^{a}-1\right)\right) u_{0}, \quad t \in[1,2), \\
0, \quad t \in[2,+\infty) .
\end{array}\right.
$$

(iv) When $1-c\left(e^{a}-1\right) / a \neq 0$ and $e^{a}+b\left(e^{a}-1\right) / a \neq 0$ and $e^{a}+(b+c)\left(e^{a}-1\right) / a=0$, the solution of (1) is

$$
u(t)=\left\{\begin{array}{l}
\left(e^{a t}+\frac{b+c}{a}\left(e^{a t}-1\right)\right) u_{0}, \quad t \in[0,1), \\
0, \quad t \in[1,+\infty) .
\end{array}\right.
$$

(v) When $1-c\left(e^{a}-1\right) / a \neq 0$ and $e^{a}+b\left(e^{a}-1\right) / a=0$ and $e^{a}+(b+c)\left(e^{a}-1\right) / a=0$, the solution of (1) is

$$
u(t)=\left\{\begin{array}{l}
\left(e^{a t}+\frac{b+c}{a}\left(e^{a t}-1\right)\right) u_{0}, \quad t \in[0,1), \\
0, \quad t \in[1,+\infty) .
\end{array}\right.
$$

For the case of $a=0$, we can discuss it in the same way.

\section{THE STABILITY OF ANALYTIC SOLUTION}

Definition 2. A solution $u(t)$ of (1) is called asymptotically stable if it satisfies $u(t) \rightarrow 0$ as $t \rightarrow \infty$.

Theorem 2. [5] The solution of (1) is asymptotically stable for any given $u_{0}$, if and only if $|\lambda|<1$, where $\lambda$ is defined in Theorem 1.

So we have the following theorem for stability.

Theorem 3. The solution of (1) is asymptotically stable for any given $u_{0}$, if any of the following conditions is satisfied:

$$
\left\{\begin{array}{l}
(a+b+c)\left(e^{a}-1\right)\left((a+b-c) e^{a}-(b-a-c)\right)<0, \\
(a+b+c)\left(e^{a}-1\right)\left((a+b+c) e^{a}-(b-a+c)\right)<0,
\end{array} \quad c \neq a /\left(e^{a}-1\right)\right.
$$


for $a \neq 0$ and

$$
(b+c)(b+2)\left(b^{2}+b c+2 b+2\right)<0, \quad c \neq 1
$$

for $a=0$.

Proof 2. According to Theorems 1 and 2 we have that the solution of (1) is asymptotically stable for any given $u_{0}$ if and only if

$$
\left|\frac{\left(e^{a}+b\left(e^{a}-1\right) / a\right)\left(e^{a}+(b+c)\left(e^{a}-1\right) / a\right)}{1-c\left(e^{a}-1\right) / a}\right|<1
$$

for $a \neq 0$ and

$$
\left|\frac{(b+1)(b+c+1)}{1-c}\right|<1
$$

for $a=0$.

If $a \neq 0$, from (10) we have the following two inequalities hold

$$
\begin{aligned}
& \left|\frac{e^{a}+b\left(e^{a}-1\right) / a}{1-c\left(e^{a}-1\right) / a}\right|<1, \\
& \left|e^{a}+\frac{b+c}{a}\left(e^{a}-1\right)\right|<1,
\end{aligned}
$$

which are equivalent to

$$
\left\{\begin{array}{l}
\left(\frac{e^{a}+b\left(e^{a}-1\right) / a}{1-c\left(e^{a}-1\right) / a}+1\right)\left(\frac{e^{a}+b\left(e^{a}-1\right) / a}{1-c\left(e^{a}-1\right) / a}-1\right)<0, \\
\left(e^{a}+\frac{b+c}{a}\left(e^{a}-1\right)+1\right)\left(e^{a}+\frac{b+c}{a}\left(e^{a}-1\right)-1\right)<0,
\end{array}\right.
$$

So we can get (8). If $a=0$, similar to the case of $a \neq 0$, we can get (9) from (11).

\section{THE OSCILLATION OF ANALYTIC SOLUTION}

Definition 3. A non-trivial solution of (1) is said to be oscillatory if there exists a sequence $\left\{t_{k}\right\}_{k=1}^{\infty}$ such that $t_{k} \rightarrow \infty$ as $k \rightarrow \infty$ and $u\left(t_{k}\right) u\left(t_{k-1}\right) \leq 0$, otherwise it is called nonoscillatory. We say (1)is oscillatory if all the non-trivial solution of (1) are oscillatory; we say (1) is non-oscillatory if all the non-trivial solutions of (1) are non-oscillatory.

Remark 2. If a solution $u(t)$ of (1) is continuous and non-oscillatory, then it must be eventually positive or negative. That is, there exists a $T \in R$ such that $u(t)$ is positive or negative for $t \geq T$.

Theorem 4. Assume that

$$
b+c<-\frac{a e^{a}}{e^{a}-1}
$$

holds, then the functional differential inequality

$$
u^{\prime}(t)-a u(t)-b u([t])-c u\left(2\left[\frac{t+1}{2}\right]\right) \leq 0
$$


has no eventually positive solution, where $a, b, c \in R$.

Proof 3. If $u(t)$ is a solution of Inequality (12) for $t \geq 2 n$, where $n$ is a sufficiently large integer. For $2 n \leq t<2 n+1$, Inequality (12) becomes

$$
u^{\prime}(t)-a u(t)-(b+c) u(2 n) \leq 0
$$

or

$$
y^{\prime}(t)-(b+c) \exp \left(\int_{2 n}^{t}(-a) d s\right) y(2 n) \leq 0
$$

where

$$
y(t)=u(t) \exp \left(\int_{2 n}^{t}(-a) d s\right) .
$$

Integrating (13) from $2 n$ to $2 n+1$, we have

$$
y(2 n+1) \leq y(2 n)\left(1+\int_{2 n}^{2 n+1}(b+c) \exp \left(\int_{2 n}^{t}(-a) d s\right) d t\right) .
$$

Since $y(t)>0$ for $t \geq 2 n$, then

$$
1+\int_{2 n}^{2 n+1}(b+c) \exp \left(\int_{2 n}^{t}(-a) d s\right) d t>0
$$

or

$$
b+c>-\frac{a e^{a}}{e^{a}-1} .
$$

This contradicts $b+c<-a e^{a} /\left(e^{a}-1\right)$. So Inequality (12) has no eventually positive solution.

Similarly, we have the following theorem.

Theorem 5. Assume that

$$
b+c<-\frac{a e^{a}}{e^{a}-1}
$$

holds, then the functional differential inequality

$$
u^{\prime}(t)-a u(t)-b u([t])-c u\left(2\left[\frac{t+1}{2}\right]\right) \geq 0
$$

has no eventually negative solution, where $a, b, c \in R$.

Theorem 6. Assume that

$$
b+c<-\frac{a e^{a}}{e^{a}-1}
$$

holds, then equation

$$
u^{\prime}(t)-a u(t)-b u([t])-c u\left(2\left[\frac{t+1}{2}\right]\right)=0
$$

has only oscillatory and non-trivial solutions, where $a, b, c \in R$. 
Proof 4. The proof can be completed by means of Theorems 4 and 5.

Theorem 7. Assume that

$$
b>-\frac{a e^{a}}{e^{a}-1}
$$

and

$$
c>\frac{a}{e^{a}-1}
$$

hold, then the Inequality (12) has no eventually negative solution.

Proof 5. Assume that $u(t)<0$ is a solution of Inequality (12) for $t \geq 2 n-1$, where $n$ is a sufficiently large integer. For $2 n-1 \leq t<2 n$, Inequality (12) gives

$$
u^{\prime}(t)-a u(t)-b u(2 n-1)-c u(2 n) \leq 0
$$

or

$$
y^{\prime}(t)-c \exp \left(\int_{2 n}^{t}(-a) d s\right) y(2 n)-b e^{-a} \exp \left(\int_{2 n}^{t}(-a) d s\right) y(2 n-1) \leq 0,
$$

where $y(t)$ is defined in (14).

Integrating (17) from $2 n-1$ to $2 n$, we have

$$
\begin{aligned}
& y(2 n)\left(1+\int_{2 n-1}^{2 n}(-c) \exp \left(\int_{2 n}^{t}(-a) d s\right) d t\right) \\
\leq & y(2 n-1)\left(1+\int_{2 n-1}^{2 n} b e^{-a} \exp \left(\int_{2 n}^{t}(-a) d s\right) d t\right),
\end{aligned}
$$

which implies that

$$
y(2 n)\left(1-\frac{c}{a}\left(e^{a}-1\right)\right) \leq y(2 n-1)\left(e^{a}+\frac{b}{a}\left(e^{a}-1\right)\right) .
$$

Since $y(t)<0$ for $t \geq 2 n-1$, then we have (i) If $b>-a e^{a} /\left(e^{a}-1\right)$ then $c<a /\left(e^{a}-1\right)$, which contradicts $c>a /\left(e^{a}-1\right)$. (ii) If $c>a /\left(e^{a}-1\right)$, then $b<-a e^{a} /\left(e^{a}-1\right)$, which is $a$ contradiction to $b>-a e^{a} /\left(e^{a}-1\right)$.

Therefore, Inequality (12) has no eventually negative solution.

Similarly, we have the following result.

Theorem 8. Assume that

$$
b>-\frac{a e^{a}}{e^{a}-1}
$$

and

$$
c>\frac{a}{e^{a}-1}
$$

hold, then the Inequality (15) has no eventually positive solution. 
Theorem 9. Assume that

$$
b>-\frac{a e^{a}}{e^{a}-1}
$$

and

$$
c>\frac{a}{e^{a}-1}
$$

hold, then the Equation (16) has only oscillatory and non-trivial solutions.

Proof 6. Follows directly from Theorems 7 and 8, the proof can be completed.

Theorem 10. Assume that

$$
b<-\frac{a e^{a}}{e^{a}-1}, \quad c<\frac{a}{e^{a}-1}, \quad b+c>-\frac{a e^{a}}{e^{a}-1}
$$

hold, then the Equation (16) has only oscillatory and non-trivial solutions.

Proof 7. Without loss of generality, we assume that $u(t)>0$ is a solution of (16) for $t \geq 2 n-1$, where $n$ is a sufficiently large integer. According to the proof of Theorem 7 , for $2 n-1 \leq t<2 n$, (16) yields

$$
y(2 n)\left(1-\frac{c}{a}\left(e^{a}-1\right)\right)=y(2 n-1)\left(e^{a}+\frac{b}{a}\left(e^{a}-1\right)\right),
$$

where $y(t)$ is defined in (14).

Since $y(t)>0$ for $t \geq 2 n-1$, then we have $(i) b \geq-a e^{a} /\left(e^{a}-1\right)$ and $c \leq a /\left(e^{a}-1\right)$, (ii) $b \leq-a e^{a} /\left(e^{a}-1\right)$ and $c \geq a /\left(e^{a}-1\right)$, which contradicts the condition (18). The proof is finished.

In view of Theorem 1 we get the following result.

Corollary 1. Assume that $a \neq 0$ and

$$
\lambda=\frac{\left(e^{a}+b\left(e^{a}-1\right) / a\right)\left(e^{a}+(b+c)\left(e^{a}-1\right) / a\right)}{1-c\left(e^{a}-1\right) / a} \neq 0,
$$

then (1) has a unique solution on $[0, \infty)$

$$
u(t)=\left\{\begin{array}{l}
e^{a(t-2 n+1)} c_{2 n-1}+\frac{1}{a}\left(e^{a(t-2 n+1)}-1\right)\left(b c_{2 n-1}+c c_{2 n}\right), \quad t \in[2 n-1,2 n), \\
\left(e^{a(t-2 n)}+\frac{b+c}{a}\left(e^{a(t-2 n)}-1\right)\right) c_{2 n}, \quad t \in[2 n, 2 n+1),
\end{array}\right.
$$

where the sequence $\left\{c_{n}\right\}$ satisfies the difference equation

$$
\begin{aligned}
& c_{2 n}=\frac{e^{a}+b\left(e^{a}-1\right) / a}{1-c\left(e^{a}-1\right) / a} c_{2 n-1}, \quad n=1,2, \cdots \\
& c_{2 n+1}=\left(e^{a}+\frac{b+c}{a}\left(e^{a}-1\right)\right) c_{2 n}, \quad n=0,1,2, \cdots,
\end{aligned}
$$

where $c_{n}=u(n)$ and $n \in N$. 
Lemma 1. Function

$$
g(y)=-a e^{a y} /\left(e^{a y}-1\right)
$$

is strictly monotonic increasing on $(0,1]$, where $a \neq 0$.

Lemma 2. Inequality $b+c>-a e^{a y} /\left(e^{a y}-1\right)$ holds on $(0,1)$ implies that

$$
e^{a y}+\frac{b+c}{a}\left(e^{a y}-1\right)>0
$$

where $a \neq 0$.

Theorem 11. Assume that $a \neq 0$ and $\lambda \neq 0$, then the Equation (16) is oscillatory if and only if any of the following conditions is satisfied:

$$
\left\{\begin{array}{l}
b>-\frac{a e^{a}}{e^{a}-1}, \quad c>\frac{a}{e^{a}-1}, \\
b<-\frac{a e^{a}}{e^{a}-1}, \quad c<\frac{a}{e^{a}-1}, \quad b+c>-\frac{a e^{a}}{e^{a}-1}, \\
b+c<-\frac{a e^{a}}{e^{a}-1} .
\end{array}\right.
$$

Proof 8. Sufficiency. It follows from (20) that the sequence $\left\{c_{n}\right\}$ oscillates under any of the condition (21). Since $u(n)=c_{n}$ for $n \in N$, so $u(t)$ also oscillates.

Necessity. we assume that any of the following hypotheses is satisfied:

$$
\left\{\begin{array}{lll}
b<-\frac{a e^{a}}{e^{a}-1}, & c>\frac{a}{e^{a}-1}, \quad b+c>-\frac{a e^{a}}{e^{a}-1}, \\
b>-\frac{a e^{a}}{e^{a}-1}, & c<\frac{a}{e^{a}-1}, \quad b+c>-\frac{a e^{a}}{e^{a}-1} .
\end{array}\right.
$$

Let $u(t)$ be the solution of (16) with $u(0)=u_{0}$, then from (20) and (22) we have $c_{n}>0$ for $n=0,1,2, \cdots$. By Corollary 1, Lemmas 1 and 2 we get

(i) For $2 n \leq t<2 n+1$ and $n \in N$,

$$
u(t)=\left(e^{a(t-2 n)}+\frac{b+c}{a}\left(e^{a(t-2 n)}-1\right)\right) c_{2 n}>0 .
$$

(ii) For $2 n-1 \leq t<2 n$ and $n \in N$,

$$
\begin{aligned}
u(t) & =e^{a(t-2 n+1)} c_{2 n-1}+\frac{1}{a}\left(e^{a(t-2 n+1)}-1\right)\left(b c_{2 n-1}+c c_{2 n}\right) \\
& =\left(c_{2 n-1}+\frac{b}{a} c_{2 n-1}+\frac{c}{a} c_{2 n}\right) e^{a(t-2 n+1)}-\frac{b}{a} c_{2 n-1}-\frac{c}{a} c_{2 n} .
\end{aligned}
$$

So we know that $u(t)$ is a monotonous function on the interval $[2 n-1,2 n)$. $u(t)$ is left continuous at the point $t=2 n$, so $u(t)$ has the minimum value $u(2 n)$ or $u(2 n-1)$ on the interval $[2 n-1,2 n)$, which implies that

$$
u(t) \geq \min \{u(2 n-1), u(2 n)\}>0 .
$$

Combining (i) and (ii), we obtain $u(t)>0, t \in[2 n-1,2 n+1)$ for $n \in N$. This contradicts the assumption that $u(t)$ oscillates. The proof is complete. 


\section{ACKNOWLEDGEMENTS}

This work is supported by the National Natural Science Foundation of China (No. 11201084) and Natural Science Foundation of Guangdong Province (No. 2017A030313031).

\section{REFERENCES}

[1] M.C. Pullan, Linear optimal control problems with piecewise analytic solutions, J. Math. Anal. Appl. 197(1996), 207-226.

[2] J. Wiener, Differential equations with piecewise constant delays, in: V. Lakshmikantham (Ed.), Trends in the Theory and Practice of Nonlinear Differential Equations, Marcel Dekker, New York, 1983, pp. $547-552$.

[3] S.M. Shah, J. Wiener, Advanced differential equations with piecewise constant argument deviations, Int. J. Math. Math. Sci. 6(1983), 671-703.

[4] K.L. Cooke, J. Wiener, Retarded differential equations with piecewise constant delays, J. Math. Anal. Appl. 99(1984), 265-297.

[5] J. Wiener, Generalized Solutions of Functional Differential Equations, World Scientific, Singapore, 1993.

[6] I. Ozturk, F. Bozkurt, Stability analysis of a population model with piecewise constant arguments, Nonlinear Anal. RWA 12(2011), 1532-1545.

[7] M.S. Alwan, X.Z. Liu, W.C. Xie, Comparison principle and stability of differential equations with piecewise constant arguments, J. Franklin I. 350(2013), 211-230.

[8] C.Y. Zhang, L.N. Jiang, Remotely almost periodic solution to systems of differential equations with piecewise constant argument, Appl. Math. Lett. 21(2008), 761-768.

[9] M.U. Akhmet, Asymptotic behavior of differential equations with piecewise constant arguments, Appl. Math. Lett, 21(2008), 951-956.

[10] M.U. Akhmet, Integral manifolds of differential equations with piecewise constant argument of generalized type, Nonlinear Anal. TMA 66(2007), 367-383.

[11] Y. Muroya, New contractivity condition in a population model with piecewise constant arguments, J. Math. Anal. Appl. 346(2008), 65-81.

[12] H. Bereketoglu, M. Lafci, G.S. Oztepe, On the oscillation of a third order nonlinear differential equation with piecewise constant arguments, Mediterr. J. Math. 14 (2017), Article ID 10.1007/s00009-017-0923-9, 19 pages.

[13] M.Z. Liu, M.H. Song, Z.W. Yang, Stability of Runge-Kutta methods in the numerical solution of equation $u^{\prime}(t)=a u(t)+a_{0} u([t])$, J. Comput. Appl. Math. 166(2004), 361-370.

[14] M.H. Song, M.Z. Liu, Numerical stability and oscillation of the Runge-Kutta methods for the differential equations with piecewise continuous arguments alternately of retarded and advanced type, J. Inequal. Appl. 2012(2012), Article ID 10.1186/1029-242X-2012-290, 13 pages. 